\title{
Effects of percentage of shock avoidance on avoidance behavior in gerbils
}

\author{
PETER F. GALVANI \\ State University of New York, College at Brockport, Brockport, New York 14420
}

\begin{abstract}
Gerbils received either $100 \%, 50 \%$, or $0 \%$ shock avoidance following shuttle responding in discriminative avoidance acquisition. Response frequency in acquisition and percentage of reinforcement were positively related. Percentage of reinforcement did not appreciably influence resistance to extinction. The results were discussed in terms of the importance of the shock-avoidance contingency, relative to other contingencies, in reinforcing the avoidance response.
\end{abstract}

In order to investigate the effects of intermittent reinforcement on discriminative avoidance learning, one must specify the sources of reinforcement for the avoidance response. As many as four sources-US escape, US avoidance, CS termination, and feedback-might be regarded as potential facilitators of avoidance response strength. Previous studies of intermittent reinforcement in the author's laboratory (Galvani, 1971, 1973a, b; Galvani, Twitty, \& Foster, 1975) have employed a "classical trial" to define nonreinforcement. Procedurally classical trials eliminate, or at least attenuate, all of the above noted sources of reinforcement for the avoidance response. Thus, on classical trials the avoidance response does not terminate the CS, the US is not avoidable, and the amount of response-produced feedback is reduced in that no stimulus change occurs consequent on the response. Since the studies cited above used a fixed-duration .5-sec shock, the US-escape contingency was also minimized. (Apparently the .5 -sec shock duration, however, does provide considerable opportunity for adventitiously reinforced escape as reported in Galvani, 1974.)

Although the classical trial provides a defensible specification of a nonreinforced trial in the avoidance paradigm, a variety of alternative nonreinforcement procedures are available (see Katzev \& Enkema, 1973). The present experiment concerns one such alternative to the classical-trial nonreinforcement procedure. On nonreinforced trials, the avoidance response resulted in CS termination, hence response-produced feedback, but no US avoidance. The effects of varying percentage of reinforcement, i.e., US avoidance, on acquisition and resistance to extinction of shuttle avoidance in gerbils was studied. An additional partial reinforcement group, comparable to those of previous studies conducted in the author's laboratory, was also included in the experiment.

The research reported in this paper was supported by Grant B04355-001 from the National Science Foundation, awarded to the author.

\section{METHOD}

\section{Subjects}

The subjects were 50 adult male gerbils obtained from Tumblebrook Farms Inc., West Brookfield, Massachusetts. All gerbils were individually housed in a continuously illuminated room and maintained on ad lib food and water throughout the experiment.

\section{Apparatus}

The apparatus consisted of two Scientific Prototype Model A100S toggle-floor shuttleboxes (13.5 x $13 \times 40 \mathrm{~cm})$ described in detail in Galvani (1971). Each shuttlebox was enclosed in a ventilated, sound-attenuated chamber illuminated by a $7.5-\mathrm{W}$ incandescent lamp. A continuous masking noise, as well as the tonal CS $(2,500 \mathrm{~Hz})$, was delivered through an $8-\mathrm{cm}$ speaker centered above the shuttlebox by a BRS-Foringer audio generator (AU-902). The tone raised the sound level ( $84 \mathrm{~dB}$ with masking noise and ventilating fan on) $2 \mathrm{~dB}$ on the $\mathrm{C}$ scale of a General Radio Type 1561-A sound-level meter. The US was a $.5-\mathrm{mA}$ (nominal) $.5-\mathrm{sec}$ scrambled electric shock delivered to the shuttlebox grids by a Grason-Stadler Model GS 700 shock generator. Programming and data recording were accomplished by electromechanical equipment located adjacent to the soundattenuated chambers.

\section{Procedure}

All gerbils received 4 days of acquisition and 2 days of extinction in 50-trial sessions with an average ITI of $30 \mathrm{sec}$ A 5-min habituation period in the shuttlebox preceded each daily session. The CS-US interval in acquisition was $5 \mathrm{sec}$, with the .5 -sec shock delivered coincident with CS offset.

There were four groups $(n=10$ each) in the experiment. Group AV100 received $100 \%$ reinforcement, i.e., on all acquisition trials a shuttle response (CR) made during the CS terminated the tone immediately and precluded the US. There were two partial reinforcement (PR) groups. Group PR100 had immediate CS termination following each $\mathrm{CR}$, but shock occurred $5 \mathrm{sec}$ after CS onset, on a random 50\% schedule following CRs. Group PR50 received both CS termination and US avoidance following a CR on a random $50 \%$ schedule, while on the remaining $50 \%$ of the trials the CS was not terminated and shock occurred at the end of the CS-US interval. Both PR groups, therefore, had a $50 \%$ reinforcement schedule with respect to US avoidance. The PR50 group, however, received nonreinforced trials of the classical type, as in previous studies (e.g., Galvani, 1971). Finally, Group CC100 gerbils experienced CS termination after every CR, but on all trials CS onset was inevitably followed by shock (hence $0 \%$ reinforcement). Failure to respond in acquisition resulted in shock for all subjects. 


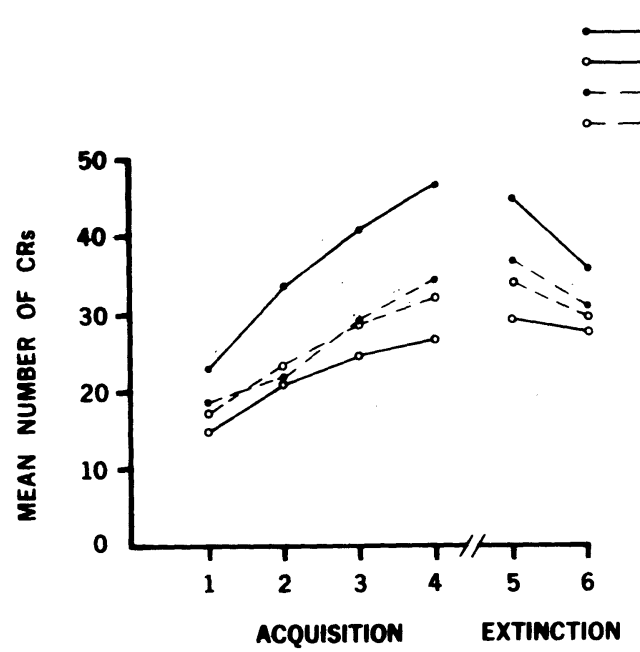

50 TRIAL BLOCKS

Figure 1. Mean number of CRs for each group of gerbils over four blocks of acquisition and over two extinction trial blocks.

All animals received the same extinction treatment, in which no shocks were delivered. A CR terminated the CS immediately, and if no response occurred the CS automatically terminated $5 \mathrm{sec}$ after its onset.

\section{RESULTS}

The mean number of CRs in both acquisition and extinction is shown for all four groups in Figure 1. The CR measure refers to any shuttle response that occurred in the CS-US interval, regardless of whether or not the response avoided shock. The mean acquisition curves reveal that, while the two PR groups were essentially the same in performance, the PR animals achieved a level of responding intermediate between continuously reinforced gerbils (AV100) and those subjects that had $0 \%$ reinforcement in terms of US avoidance (CC100). An ANOVA of the acquisition data of Groups AV100, PR100, and CC100 revealed that the effects of both percentage of US avoidance $[F(2,27)=12.73, p<.001]$, and trial blocks $[\mathrm{F}(3,81)=29.02, \mathrm{p}<.001]$ were reliable, but not their interaction. While Group AV100 was superior to PR100 on Trial Block $4[t(18)=3.33$, $\mathrm{p}<.01]$, the superiority of Group PR100 to $\mathrm{CC} 100$ on Block 4 was statistically marginal $[\mathrm{t}(18)=1.88, \mathrm{p}<.1]$. Thus, when nonreinforcement in avoidance acquisition is based solely on the elimination of the shock-avoidance contingency, intermittent reinforcement results in performance inferior to that obtained under continuous reinforcement, as previously found employing the classical trial as a nonreinforcement procedure (e.g., Galvani, 1971).

Group PR50 was excluded from the previous analyses, since this group did not have the same CS termination and feedback contingencies as the other three groups. Although these animals experienced a $50 \%$ US avoidance schedule, as did Group PR100, the latter obtained $100 \%$ CS termination following CRs, rather than $50 \%$ as in Group PR50. As Figure 1 shows, despite the different CS termination contingencies, the PR groups did not differ at any point during acquisition.

All groups showed considerable CR persistence in extinction. Indeed, only Group AV100 was reliably inferior in performance on Day 6 relative to the final day of acquisition [ $t(9)=2.62, p<.05$ ] (for the other three groups, all ts $<1$ ). As in acquisition, the two PR groups performed comparably in extinction.

Since the shock avoidance contingency produced reliable acquisition differences, an extinction ratio (total CRs on extinction trial block/total CRs on acquisition Trial Block 4) was computed for both extinction sessions for all subjects in Groups AV100, PR100, and CC100 in order to evaluate relative degree of extinction between groups. The ANOVA of these ratios showed only the effect of trial blocks was reliable $[F(1,27)=11.43, p<.01]$. The percentage of US avoidance experienced in acquisition, therefore, did not significantly influence relative degree of extinction between groups.

\section{DISCUSSION}

These acquisition results clearly reveal the importance of the shock avoidance contingency in determining shuttlebox avoidance performance in the Mongolian gerbil. The results further suggest that the decremental effects of intermittent reinforcement previously observed in studies of gerbil avoidance leaming (Galvani, $1971,1973 a, b, 1975)$ were principally related to the failure to avoid the US on a nonreinforced trial, rather than being attributable to a lack of CS termination or feedback, as suggested in Galvani (1973a). Thus, in spite of receiving $100 \%$ reinforcement with respect to the CS termination and feedback contingencies, Groups AV100, PR100, and CC100, exposed to $100 \%, 50 \%$, and $0 \%$ US avoidance, respectively, differed considerably in acquisition performance. Moreover, Groups PR100 and PR50, both of which had a 50\% US avoidance schedule, were essentially indistinguishable in performance despite different CS termination contingencies.

The latter comparison does not necessarily mean, however, that CS termination and feedback contingencies were of trivial consequence under all conditions. Group CC100 showed improvement in CR strength during acquisition in the complete absence of US avoidance. Presumably, the mechanisms of CR strengthening in this group must be due to the CS termination and feedback contingencies. In this regard, previous evidence (e.g., Galvani, 1971) clearly shows that if all trials involve no CS termination as well as no US avoidance, then a decline in CR strength over trials is observed. 
Although a potent determinant of shuttlebox avoidance acquisition in gerbils, the percentage of shock avoidance received in acquisition appeared to have no effect on extinction performance. The fact that only Group AV100 showed reliable extinction below its acquisition asymptote, however, provides indirect evidence of less CR persistence following continuous reinforcement relative to intermittent reinforcement. The failure to obtain extinction differences might be limited to the extinction procedure employed, maintaining CS termination in the absence of shock, since relative differences in CR persistence have been shown to be a function of extinction procedures as well as acquisition treatments (Galvani, 1971, 1973a, b; Katzev \& Enkema, 1973).

\section{REFERENCES}

Galvani, P. F. The effects of partial reinforcement on the acquisition and extinction of avoidance behavior in gerbils. Psychonomc Science, 1971, 24, 242-244.
Galvani, P. F. Effects of US-presentation and CS-termination contingencies on avoidance extinction following partial and continuous reinforcement. Learning and Motivation, 1973, 4, 197-206. (a)

Galvani, P. F. Punished and classical-trial extinction of avoidance in gerbils following partial and continuous reinforcement. Psychological Reports, 1973, 32, 723-729. (b)

Galvani, P. F. Role of unconditioned stimulus escape and avoidance in discriminative avoidance learning in the gerbil (Meriones unguiculatus). Journal of Comparative and Physiological Psychology, 1974, 86, 846-852.

Galvani, P. F., Twitty, M. T., \& Foster, K. M. Intermittent reinforcement and intertrial interval effects on shuttlebox avoidance in the gerbil. Bulletin of the Psychonomic Society, $1975,5,225-227$.

Katzev, R. D., \& Enkema, S. J. Acquisition and extinction of signaled avoidance as a function of intermittent reinforcement. Learning and Motivation, 1973, 4, 176-196.

(Received for publication February 19, 1976.) 